\title{
Anogenital Distance
}

National Cancer Institute

\section{Source}

National Cancer Institute. Anogenital Distance. NCI Thesaurus. Code C124276.

A measurement of the length of the span between the anus and the base of the genitalia. 